\title{
Special Issue of Minds and Machines on Causality, Uncertainty and Ignorance
}

\author{
Rolf Haenni · Stephan Hartmann
}

Published online: 27 October 2006

(C) Springer Science+Business Media B.V. 2006

In everyday life, as well as in science, we have to deal with and act on the basis of partial (i.e. incomplete, uncertain, or even inconsistent) information. This observation is the source of a broad research activity from which a number of competing approaches have arisen. There is some disagreement concerning the way in which partial or full ignorance is and should be handled. The most successful approaches include both quantitative aspects (by means of probability theory) and qualitative aspect (by means of graphical or causal models or logic). Some of these approaches have important impacts on various disciplines including philosophy, computer science, statistics, mathematics, physics, and social science. Most notably, the relation between causal and probabilistic information is a topic of interest in all of these fields.

The general question of how uncertainty is related to ignorance, and the connection thereof to causality, have been the key topics of the international summer school at the University of Konstanz, Germany, in August 2004. This was the third event in a series of summer schools organized by the interdisciplinary research group "Philosophy, Probability, and Modeling". The group was in operation from September 1st, 2001, until August 31, 2005. The first summer school, held in September 2002 , focused on the application of probability theory to topics of philosophical interest. Problems and questions that arise when probabilistic models and techniques are being applied in the special sciences were then discussed during the second summer school, held in July 2003. The fourth and final event in the series, in August 2005, was devoted to philosophical questions related to the application and

\footnotetext{
R. Haenni ( $\square)$

Institute of Computer Science and Applied Mathematics,

University of Berne, Neubrückstrasse 10, Berne CH-3012, Switzerland

e-mail: haenni@iam.unibe.ch

\section{S. Hartmann}

Department of Philosophy, Logic and Scientific Method, London School of Economics and Political Science, Houghton Street, London WC2A 2AE, England e-mail: S.Hartmann@lse.ac.uk
} 
interpretation of probabilities in physics. Each of the four summer schools attracted 40-50 people from many different countries. It was an inspiring forum for an interdisciplinary discussion among researchers and students from many different areas.

This special issue collects a selection of the invited and contributed papers presented at the summer school in 2004. We acknowledge the generous support of the Center for Junior Research Fellows at the University of Konstanz, the Gesellschaft für analytische Philosophie e.V., the Centre for Philosophy of Natural and Social Science at the London School of Economics, the Alexander von Humboldt Foundation, the Federal Ministry of Education and Research and the Program for the Investment in the Future (ZIP) of the German Government through a Sofja Kovalevskaja Award. We thank the referees of the papers for their work, and James Moor for his interest in dedicating a special issue of this journal to Causality, Uncertainty and Ignorance. 